\title{
NUMERICAL STUDY OF DEFORMATION DROPS OF WATER IN GAS STREAM
}

\author{
Anna Shebeleva ${ }^{1, *}$, and Mikhail Chernetskiy ${ }^{1}$ \\ ${ }^{1}$ Siberian Federal University, Krasnoyarsk, Russia
}

\begin{abstract}
Mathematical model developed on basis of experimental data about processes of deformation organohydrocarbon drops of fuel in stream of oxidant. Numerical investigation of integral characteristics of deformation of droplet organohydrocarbon fuel with size of drops $-4.5 \mathrm{~mm}$, speed of oxidant $-2 \mathrm{~m} / \mathrm{s}$, temperature of $298 \mathrm{~K}$ has been completed (in accordance with the conditions of the experiment). Specific change of surface longitudinal and cross section and of organohydrocarbon drops of fuel during its gravity precipitation in time has been shown. It showed satisfactory agreement theoretical and experimental data. The developed model is the basis for solving problem of collapse (crushing) of drops of coal-water and organohydrocarbon slurry during their motion in a gaseous medium at a subsonic speed.
\end{abstract}

\section{Introduction}

Behavior form droplets in the gas stream is important for practical applications is pleased, for example in the field of medical diagnostics, agriculture, improvement and optimization of burners nozzles, inkjet printing, etc.

Exists numerous publications which are devoted, for example, to the investigation of droplet formation, their deformation, breakage, collisions, and evaporation [1-4]. Continuous change of shape and area occurs at the droplet surface drops deformation in flight. Therefore, the integral characteristics of heat and mass transfer between the droplet surface and the surrounding air are changed.

The review [5], published on the use of numbers only Weber and Reynolds does not allow to fully describe the patterns of strain drops. Main characteristics of the "deformation cycles" drops depend on the initial droplet size, droplet velocity, gas temperature and fluid properties (density, viscosity and surface tension). This feature should be considered in simulating the deformation of droplets in the gas stream.

The aim of this study was to: testing of numerical methods and research of features of deformation processes of water droplets as they move into a gas stream (air), which takes into account the impact of the initial droplet size, velocity, liquid properties and gaseous medium.

\footnotetext{
* Corresponding author: an riv@mail.ru
} 


\section{Mathematical model}

Numerical methods are used in this paper to simulate two-phase flows in a T-microchannel, based on the volume of fluid method in the cells. This works well for the calculation of macroscopic flows with free surface [6-9]. The idea of the method is that the liquid and gas are treated as a single two-component medium and the spatial distribution of the phases within the computational domain is determined using a marker function $F(x, y, z, t$,$) , which value specifies$ the volume fraction of the liquid phase in the computational cell as follows:

$$
F(x, y, z)=\left\{\begin{array}{l}
0, \text { if the cell is empty } \\
1, \text { if the cell is completely filled with the liquid }
\end{array}\right.
$$

$0<F(x, y, z, t)<1-$ if the phase boundary passes through the cell.

Since the free surface moves together with the liquid, tracking of the free boundary in space is performed by solving the transport equation for the volume fraction of the liquid phase in the cell:

$$
\frac{\partial F}{\partial t}+V \cdot \nabla F=0
$$

Here $\boldsymbol{V}$ is the velocity vector of the two-phase medium obtained by solving the hydrodynamic equations, consisting of the equations of conservation of mass, or the continuity equation:

$$
\frac{\partial \rho}{\partial t}+\nabla(\rho \boldsymbol{V})=0
$$

and the equations of motion and the law of conservation of momentum:

$$
\frac{\partial \rho V}{\partial t}+\nabla \frown \rho V \times V=-\nabla p+\nabla \frown T \mp F
$$

Where $\tau$ - viscous stress tensor, $\boldsymbol{F}$ - vector of body forces, p - static pressure, $\rho$ - the density of the two-phase medium.

The components of the viscous stress tensor $\tau_{i j}$ defined as:

$$
\tau_{i j}=\mu\left(\frac{\partial U_{i}}{\partial x_{j}}+\frac{\partial U_{j}}{\partial x_{i}}-\frac{2}{3} \delta_{i j} \frac{\partial U_{k}}{\partial x_{k}}\right)
$$

Where $\mu$ - viscosity coefficient of the liquid, $U_{i j}$ - the components of the velocity vector.

The density and viscosity coefficient of the two-component medium considered obtained from the volume fraction of liquid in the cell:

$$
\begin{aligned}
& \rho=\rho_{1} F+(1-F) \mu_{2} \\
& \mu=\mu_{1} F+(1-F) \mu_{2}
\end{aligned}
$$


Here, $\rho 1, \mu 1$ - density and viscosity coefficient of the liquid, $\rho 2, \mu 2$ - the density and viscosity coefficient of the second fluid.

Thus obtained density values $\rho$ and viscosity $\mu$ are included in the equation of motion and determine the physical properties of the two-phase medium. The phenomenon of surface tension occurs when considering the fluid flows with the interface, which cannot be ignored in the case of flow in microchannels. Surface tension plays a key role in microchannels. The study of currents, controlled by surface tension, is a very difficult task. VOF method makes it relatively easy to take into account the effect of surface tension forces. Most often, the VOF method uses the so-called continuum surface force (CSF) algorithm to model surface tension $[10,11]$. The essence of this algorithm is that the equations of motion include an additional volume force $F_{s}$ whose magnitude is given by the relation:

$$
F_{S}=\sigma k \nabla F
$$

Where $\sigma$ is the surface tension coefficient and $k$ is the curvature of the free surface determined by the divergence of the normal vector:

$$
k=\nabla\left(\frac{n}{|n|}\right)
$$

The normal to the free surface is in turn calculated as the gradient of the volume fraction of the liquid phase in the cell:

$$
n=\nabla F
$$
$\theta$ :

On the solid wall, the magnitude of the normal vector is determined from the contact angle

$$
n=n_{w} \cos \theta+\tau_{w} \sin \theta
$$

Where $n_{w}, \tau_{w}$ - are the normal and tangential vectors to the wall.

\section{Formulation of the problem. Results}

In this study, a numerical method of calculation of two-phase flows with a free surface was tested. Experimental data were taken from a paper [5]. The geometry of the task is shown in figure 1. Geometry is a rectangle with a tube from which the water flows at a speed of $0.12 \mathrm{~m} /$ s. axisymmetric formulation used. The inner diameter of the nozzle of the dispenser is $2.5 \mathrm{~mm}$.

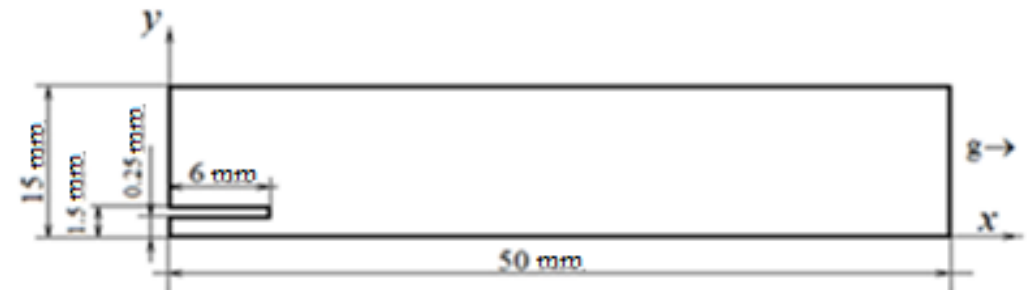

Fig. 1. Geometry of the case.

A drop of water moving with a certain "deformation cycles" sequence. The complexity is the simulation of the formation of the expiry of the jet of droplets from a nozzle at the initial 
stage. Nozzle geometry, fluid dispensing speed and the contact angle must be taken into account for an adequate description of this process.

Comparison of simulation results with experimental data [5] presented in figure 2 at the stage of the formation of drops. As can be seen, the agreement between calculation and experiment shows good dynamics for the formation of drops and form of the free surface.
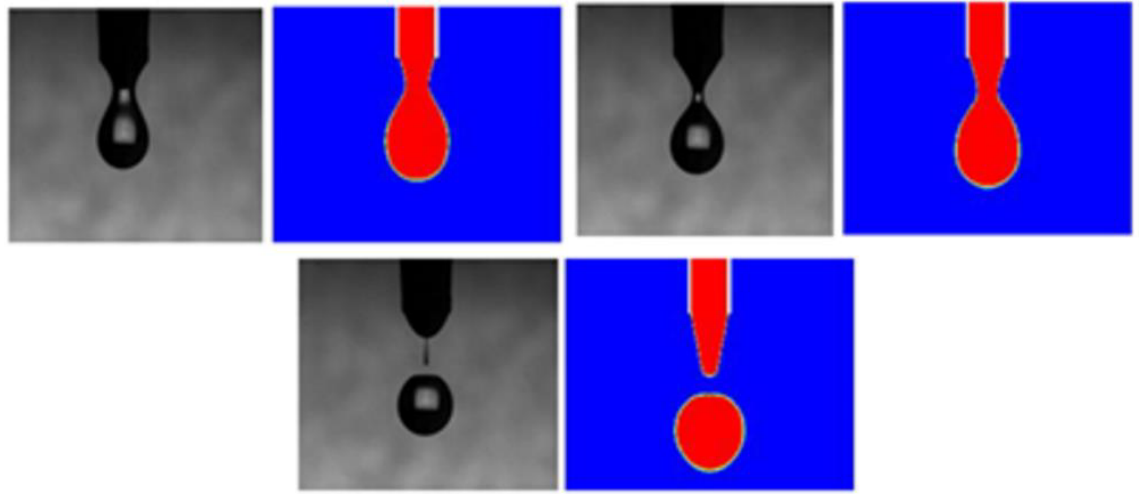

Fig. 2. Evolution of behavior of drop at initial stage. Black and white image - experiment, color images the simulation results.

Movement of droplets in the stream is accompanied by periodic oscillations of its form, after the drop formed a comparison of the dynamics of the behavior of a drop in the calculation and the experiment shown in figure 3. It may be noted, the form of droplets in the experiment and the calculation agrees well.

Comparison of the calculated drop shape with experimental photographs shown in figure 4. at various times .we visualize droplet sectional while the experiment is presented in a three-dimensional droplet shape in perspective. However, in the main points, the calculated drop shape similar to experimental photos.
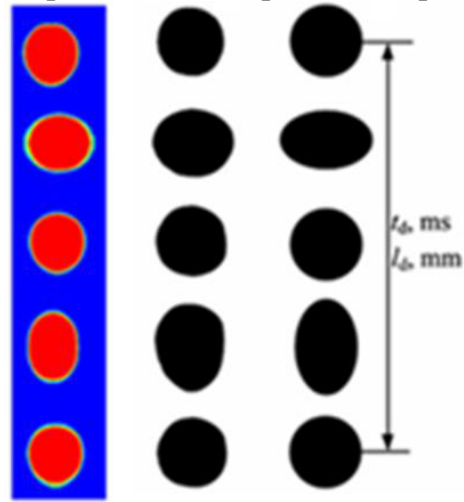

Fig. 3. Typical shapes of water drops a) The results of the calculation; $b$ ) The actual shape of the drop [5]; c) The ideal form of drops [5].
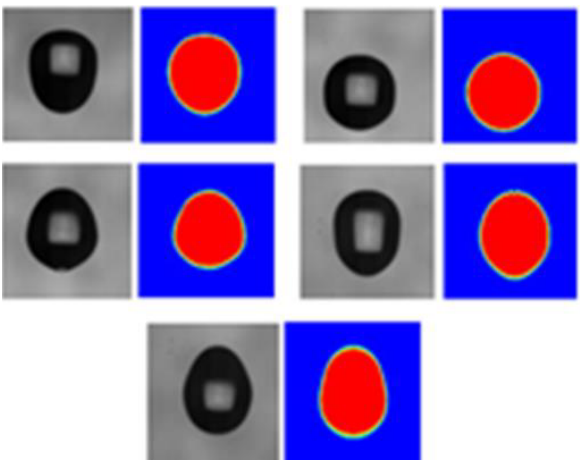

Fig. 4. Form of droplet surface in calculation and experiment at various time. Black and white image - experiment, color images - the simulation results.

Quantitative comparison of calculation and experiment is shown in figure 5. The graph shows the behavior of the relative amplitude of the vertical oscillations drops in time, related to the period of oscillations. As can be seen, the oscillation amplitude decays with time. The frequency and amplitude of the oscillations in the same experiment and calculation. 


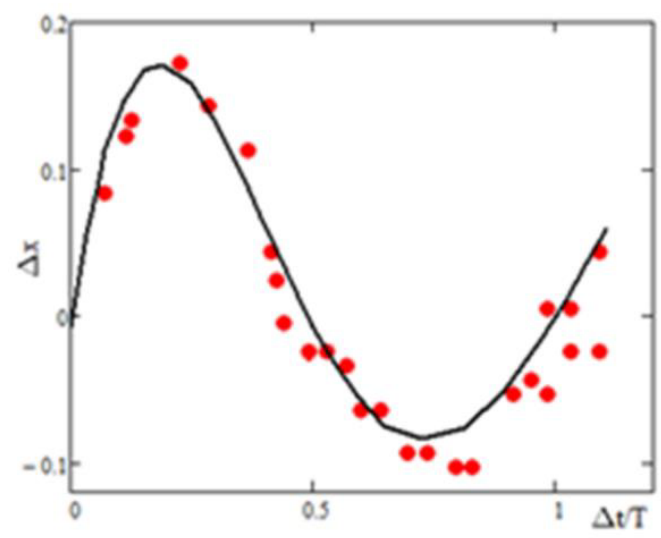

Fig. 5. Dependence of the relative vertical oscillation amplitude drops in the stream. Red facilities calculation results.

\section{Conclusion}

Mathematical model and numerical method of calculating the deformation of droplets in the gas flow is developed. The model is based on the Volume of fluid method and algorithm CSF (continuum surface force) to calculate the surface tension. A series of calculations was performed fall and deformation of water droplets. The simulation results are compared with experimental data a satisfactory agreement. In the future, we plan to develop this model to the problem of deformation of drops coal-water slurry.

\section{Acknowledgments}

This research was done at the expense of the Russian Science Foundation (Project No. 15-1910003).

\section{References}

1. S.K. Aggarwal, F. Peng, J. Eng. Gas Turbines Power 117 (1995)

2. R.S. Volkov, A.O. Zhdanova, M.V. Zabelin, G.V. Kuznetsov, P.A. Strizhak, Eur. Phys. J. Web Conf. 76 (2014)

3. G.V. Kuznetsov, P.A. Strizhak, High Temp. 52 (2014)

4. R.S. Volkov, G.V. Kuznetsov, P.A. Strizhak, Int. J. Heat Mass Transfer. 79 (2014)

5. R. S. Volkov, G. V. Kuznetsov, P.A. Strizhak. Int. J. Heat Mass Transfer 85 (2015)

6. A.V Minakov. Comp. Math. Math. Phys. 54 (2014)

7. D.V. Guzei, A.V Minakov, M.I Pryazhnikov, A.A Dekterev, T. A., Thermophysics and Aeromechanics 22 (2015)

8. R.I Iegorov, P.A.Strizhak, M.Y. Chernetskiy. EPJ Web of Conf. 110 (2016)

9. A.V. Minakov, M.V. Pervukhin, D.V. Platonov, M.Y. Khatsayuk. Comp. Math. Math. Phys. 55 (2015)

10. A.A. Shebeleva, A.V. Minakov, A.A. Yagodnitsina, V.G. Andyuseva, MATEC Web Conf. 23 (2015)

11. A. S. Orlov, A. V. Minakov, A. V. Proshkin, MATEC Web Conf. 23 (2015) 